\title{
Controllable $0-\pi$ Josephson junctions containing a ferromagnetic spin valve
}

\author{
E. C. Gingrich ${ }^{1,2 \dagger}{ }^{\dagger}$, Bethany M. Niedzielski ${ }^{1 \dagger}$, Joseph A. Glick ${ }^{1}$, Yixing Wang ${ }^{1:}$, D. L. Miller ${ }^{2}$, Reza Loloee ${ }^{1}$, \\ W. P. Pratt Jr ${ }^{1}$ and Norman O. Birge ${ }^{1 \star}$
}

Superconductivity and ferromagnetism are antagonistic forms of order, and rarely coexist. Many interesting new phenomena occur, however, in hybrid superconducting/ferromagnetic systems. For example, a Josephson junction containing a ferromagnetic material can exhibit an intrinsic phase shift of $\pi$ in its ground state for certain thicknesses of the material'. Such ' $\pi$-junctions' were first realized experimentally in 2001 (refs 2,3 ), and have been proposed as circuit elements for both high-speed classical superconducting computing and for quantum computing ${ }^{4-10}$. Here we demonstrate experimentally that the phase state of a Josephson junction containing two ferromagnetic layers can be toggled between 0 and $\pi$ by changing the relative orientation of the two magnetizations. These controllable $0-\pi$ junctions have immediate applications in cryogenic memory, where they serve as a necessary component to an ultralow power superconducting computer"1. Such a fully superconducting computer is estimated to be orders of magnitude more energy-efficient than current semiconductor-based supercomputers ${ }^{12}$. Phase-controllable junctions also open up new possibilities for superconducting circuit elements such as superconducting 'programmable logic', where they could function in superconducting analogues to field-programmable gate arrays.

When a superconducting (S) material and a ferromagnetic (F) material are placed in contact with each other, the properties of both materials are modified near the S/F interface. The intriguing nature of this 'superconducting proximity effect' in S/F systems arises due to the exchange field in $\mathrm{F}$, which imposes a phase shift on the two electrons of a Cooper pair as they propagate across F. Cooper pairs in conventional superconductors consist of two electrons with equal and opposite momenta and opposite spin. When such a pair crosses the S/F boundary, one electron goes into the majority, or up-spin, band in $\mathrm{F}$ and the other goes into the minority, or down-spin, band, causing the two electrons to acquire a net centre-of-mass momentum $\pm \hbar Q= \pm\left(\hbar k_{\mathrm{F}}{ }^{\uparrow}-\hbar k_{\mathrm{F}}{ }^{\downarrow}\right)$, where $\hbar k_{\mathrm{F}}{ }^{\uparrow}$ and $\hbar k_{\mathrm{F}}{ }^{\downarrow}$ are the Fermi momenta of the majority and minority bands, respectively ${ }^{13}$. Alternatively, one can say that the electron pair correlation function oscillates in F with wavevector $Q$ perpendicular to the S/F interface. In S/F/S Josephson junctions, those oscillations translate into oscillations between 0 -junctions and $\pi$-junctions as the F-layer thickness is increased ${ }^{1-3}$.

Imagine now a Josephson junction with the structure $\mathrm{S} / \mathrm{F}_{1} / \mathrm{N} / \mathrm{F}_{2} / \mathrm{S}$, where $\mathrm{F}_{1}$ and $\mathrm{F}_{2}$ may be different ferromagnetic materials ${ }^{14-17}$. The pair correlation function describing Cooper pairs from the left-hand $\mathrm{S}$ accumulates a phase $\phi_{1}=Q_{1}{ }^{*} d_{\mathrm{F} 1}$ while traversing $\mathrm{F}_{1}$, where $d_{\mathrm{F} 1}$ is the thickness of $\mathrm{F}_{1}$. If the magnetization of $F_{2}$ is parallel to that of $F_{1}$, then the pair correlation function will accumulate an additional phase $\phi_{2}=Q_{2}{ }^{*} d_{\mathrm{F} 2}$ traversing $\mathrm{F}_{2}$. If, however, the magnetization of $F_{2}$ is antiparallel to that of $F_{1}$, then the role of majority and minority bands is reversed, and the pair correlation function will acquire the opposite phase, $-\phi_{2}$. As shown schematically in Fig. 1a, if we choose $\phi_{1}$ to be close to $\pi / 2$ and $\phi_{2} \leq \pi / 2$, then when the layers are parallel, $\phi=\phi_{\mathrm{P}}=\phi_{1}+\phi_{2}$, putting the junction into the $\pi$-state, and when the layers are antiparallel, $\phi=\phi_{\mathrm{AP}}=\phi_{1}-\phi_{2}$, putting the junction into the 0 -state.

Experimental verification of the prediction outlined above requires performing a phase-sensitive measurement, which we accomplish by fabricating a superconducting quantum interference device, or SQUID, containing two Josephson junctions of the structure described above. The junctions are elliptically shaped with different aspect ratios of 2.2 and 2.8 so that the magnetic layers in the two junctions will have different switching fields. We choose different ferromagnetic materials-one hard for the 'fixed layer' and the other soft for the 'free layer'-so that only the free layer switches its magnetization direction in small applied magnetic fields. The free layer was chosen as $\mathrm{Ni}_{0.84} \mathrm{Fe}_{0.16}$ ('Permalloy') of thickness $1.5 \mathrm{~nm}$ to put the junction close to the $0-\pi$ transition (J.A.G., M. A. Khasawneh, B.M.N., E.C.G., R.L., W.P.P.Jr, and N.O.B., in preparation). The fixed layer in the junctions is Ni of thickness $1.2 \mathrm{~nm}$, which should add or subtract a small phase increment ${ }^{15,18-20}$. Further information about the materials can be found in Methods. Figure $1 \mathrm{~b}$ shows a cartoon with the design of our SQUIDs and junctions, as well as the four accessible magnetic states of the junctions. We will use the figure's labelling convention for the four states as ' $\pi-\pi$ ', ' $0-\pi$ ', and so on, corresponding to the states of the two junctions, JJ-1 and JJ-2 respectively. We will show that these labels accurately describe the phase states of the junctions.

We initialize the junctions into the $\pi-\pi$ state by applying a large in-plane field of $H_{\text {in }}=-2,600 \mathrm{Oe}$, which sets all four magnetic layers in the negative direction. We then measure, at zero field, a set of $I-V$ curves with different values of the current $I_{\Phi}$ through the flux line to observe oscillations in the SQUID critical current as a function of applied flux $\Phi$. Critical currents are obtained by fitting $I-V$ curves to the standard form for an overdamped Josephson junction $^{21}$. Note that the critical currents for the two polarities of applied current, $I_{c+}$ and $I_{c-}$, need not be the same. Next we apply a small 'set' field $H_{\text {in }}=5 \mathrm{Oe}$, return the field to zero, and repeat the scan of $I-V$ curves versus flux. We continue taking small steps in $H_{\text {in }}$, each time setting the field back to zero and repeating a full flux scan. Figure 2a shows a three-dimensional plot of $I_{c+}$ versus

\footnotetext{
${ }^{1}$ Department of Physics and Astronomy, Michigan State University, East Lansing, Michigan 48824, USA. ${ }^{2}$ Northrop Grumman Systems Corporation, Baltimore, Maryland 21240, USA. †These authors contributed equally to this work. Present address: Seagate Technology, 47010 Kato Road, Fremont, California 94538, USA. *e-mail: birge@pa.msu.edu
} 


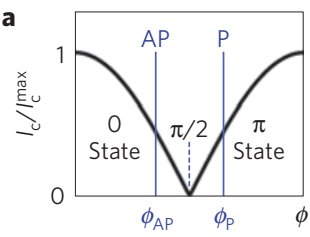

c

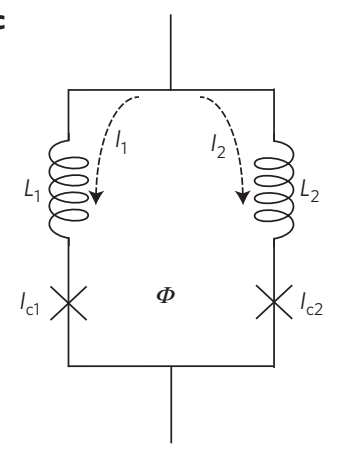

b

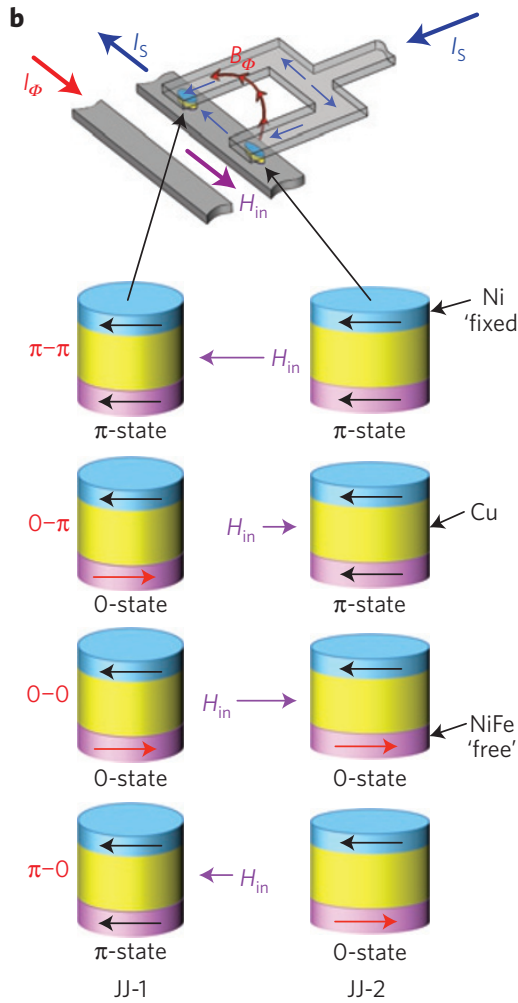

$\mathrm{JJ}-1$
Figure 1 | Schematic of experimental design. a, Cartoon showing the critical current and phase state of an $\mathrm{S} / \mathrm{F}_{1} / \mathrm{N} / \mathrm{F}_{2} / \mathrm{S}$ Josephson junction as a function of the total phase shift $\phi$ acquired by a Cooper pair traversing the entire structure. The individual phase shifts acquired through $F_{1}$ and $F_{2}$ are given in the ballistic limit by $\phi_{1}=Q_{1}{ }^{*} d_{F 1}$ and $\phi_{2}= \pm Q_{2}{ }^{*} d_{F 2}$, respectively. If $\phi_{1}=\pi / 2$ and $\phi_{2}<\pi / 2$, then the Josephson junction will be in the $\pi$-state when the magnetizations of $F_{1}$ and $F_{2}$ are parallel $(P)$, or in the 0 -state when the magnetizations are antiparallel (AP). So, by controlling the magnetic configuration of the layers in the junction, we can switch a junction between the 0 and $\pi$ state. $\mathbf{b}$, Schematic diagram of the SQUID, and cartoons showing the magnetization directions of the free and fixed layers for the four magnetic states discussed in this work. The 5 - $\mu \mathrm{m}$-wide straight bottom lead, two Josephson junctions and 5 - $\mu \mathrm{m}$-wide pitch-fork-shaped top lead make up the SQUID device, and the 10- $\mu \mathrm{m}$-wide adjacent straight long wire injects magnetic flux $\Phi$ into the SQUID loop. The inner dimensions of this loop are $10 \mu \mathrm{m}$ by $10 \mu \mathrm{m}$. The positive directions of various experimental quantities are labelled by arrows: the measurement current, $I_{s}$, the applied in-plane magnetic field, $H_{\text {in }}$, the flux-line current, $I_{\Phi}$, and the magnetic field produced by the flux line, $B_{\Phi}$. The more circular junction is labelled as ' $J J-1$ ' whereas the more eccentric elliptical junction is labelled as 'JJ-2.' The sizes of the four arrows in the four magnetic states depict the magnitude and direction of $H_{\text {in }}$ required to reach each state. $\mathbf{c}$, Basic model of an asymmetric SQUID, used for quantitative modelling of the SQUID data. $l_{1}$ and $I_{2}$ are the currents flowing through the two arms, $L_{1}$ and $L_{2}$ are the effective inductances of the two arms, and $I_{c 1}$ and $I_{\mathrm{c} 2}$ are the critical currents of the two Josephson junctions. The externally applied flux through the SQUID is $\Phi$.

$H_{\text {in }}$ and $I_{\Phi}$ as $H_{\text {in }}$ is stepped from 0 to 100 Oe. Cross-sections of the plot at fixed values of $H_{\text {in }}$ exhibit clear oscillations in $I_{c}^{+}\left(I_{\Phi}\right)$. As $H_{\text {in }}$ is varied, those cross-sections exhibit two large jumps, the first at $H_{\text {in }}=30 \mathrm{Oe}$ and the second at $H_{\text {in }}=50 \mathrm{Oe}$. At each jump, the overall magnitude of the critical current changes, and the peaks in $I_{c+}$ shift along the flux axis. We identify the first jump with the $\mathrm{NiFe}$ free layer in the more circular JJ-1 switching its magnetization direction, so that the phase state of JJ-1 switches from $\pi$ to 0 , thus changing the SQUID from the ' $\pi-\pi$ ' to the ' $0-\pi$ ' state. The second

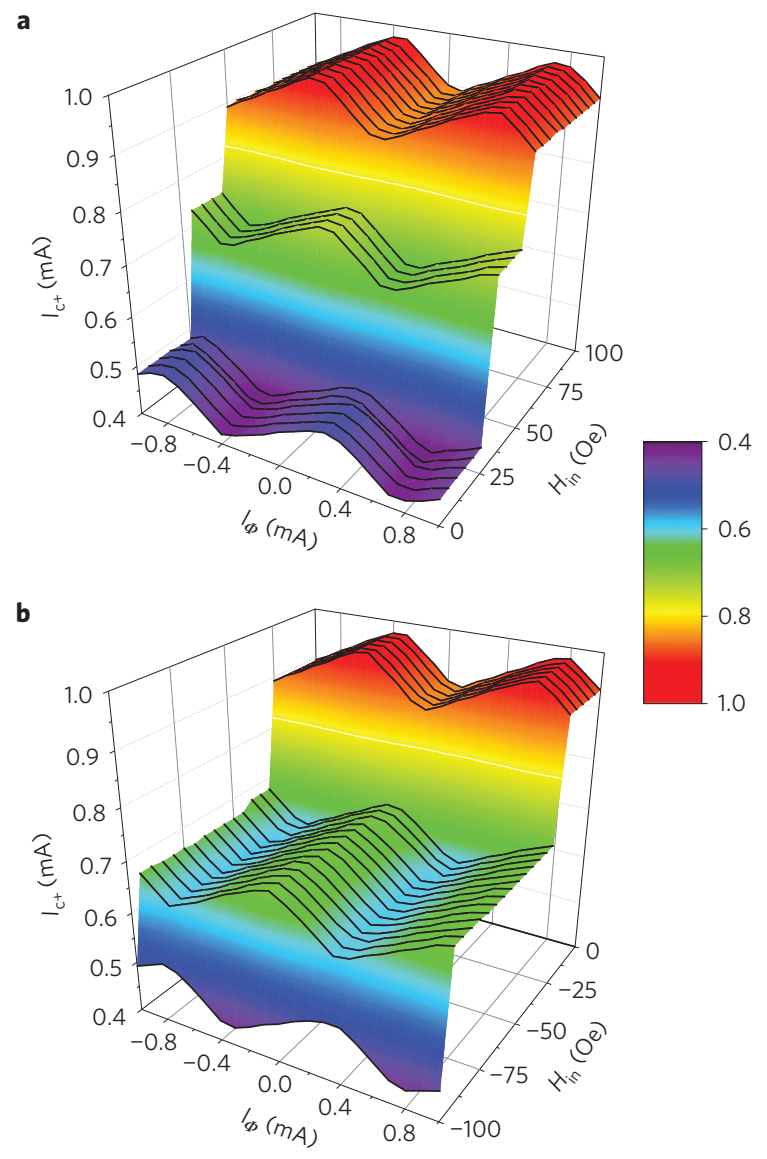

Figure 2 | Three-dimensional plots of positive SQUID critical current, $I_{\mathrm{c}+}$, versus flux-line current $I_{\Phi}$ and in-plane set field, $H_{\text {in }}$. After each value of $H_{\text {in }}$ is applied, the field is returned to zero and a scan of $I_{C+}$ versus $I_{\Phi}$ is acquired. Cross-sections at fixed $H_{\text {in }}$ show clear SQUID oscillations in $I_{C+}\left(I_{\Phi}\right)$ with a period of about $1.1 \mathrm{~mA}$, corresponding to one flux quantum $\Phi_{0}=h / 2 e$. Sudden jumps in the magnitude and phase of $I_{c+}$ indicate changes in the magnetic state of one of the Josephson junctions in the SQUID. The four total jumps cover the four magnetic states shown in the cartoon of Fig. 1b. a, Data for $H_{\text {in }}>0$. b, Data for $H_{\text {in }}<0$. In both cases the data are taken with $\left|H_{\text {in }}\right|$ increasing in time.

jump signifies that the NiFe layer in the more elliptical JJ-2 has switched its magnetization direction, and is now also in the 0-state, so the SQUID is now in the ' $0-0$ ' state. Figure $2 \mathrm{~b}$ shows similar data acquired for $H_{\text {in }}<0$. Again there are two jumps in the plot, the first occurring at $H_{\text {in }}=-35 \mathrm{Oe}$, putting the SQUID in the ' $\pi-0$ ' state, and the second at $H_{\text {in }}=-100 \mathrm{Oe}$, returning the SQUID to the ' $\pi-\pi$ ' state as at initialization. Taken together, Fig. 2a,b corresponds to a major loop through all four accessible magnetic states of the system. The fact that the magnitudes of the switching fields for $H_{\text {in }}<0$ are generally larger than for $H_{\text {in }}>0$ is due to dipolar coupling between the fixed Ni layer and the free NiFe layer in each junction.

Figure 3a shows more detailed data of $I_{\mathrm{c}+}$ and $I_{\mathrm{c}-}$ versus $I_{\Phi}$ for four selected values of $H_{\text {in }}$ taken just after each jump. Several features are immediately apparent in the data. First, $I_{c+}$ and $I_{c-}$ never approach zero; but rather oscillate with an amplitude of approximately $85 \mu \mathrm{A}$ in all four magnetic states. Second, the oscillations of $I_{c+}$ and $I_{c-}$ are not sinusoidal, but rather have an asymmetric saw tooth or ratchet shape. Third, the maxima in the $I_{c+}$ and $I_{c-}$ data do not line up with each other, so in general $I_{\mathrm{c}-}(\Phi) \neq-I_{\mathrm{c}+}(\Phi)$. All three of these features are well understood $^{21,22}$; the first is due to the finite geometrical inductance of the SQUID loop, whereas the second and third are due to 


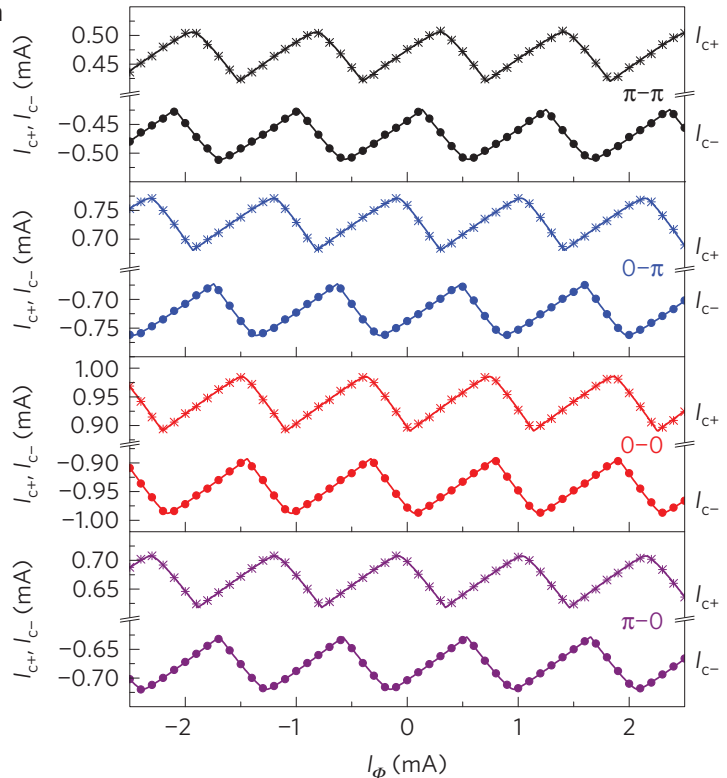

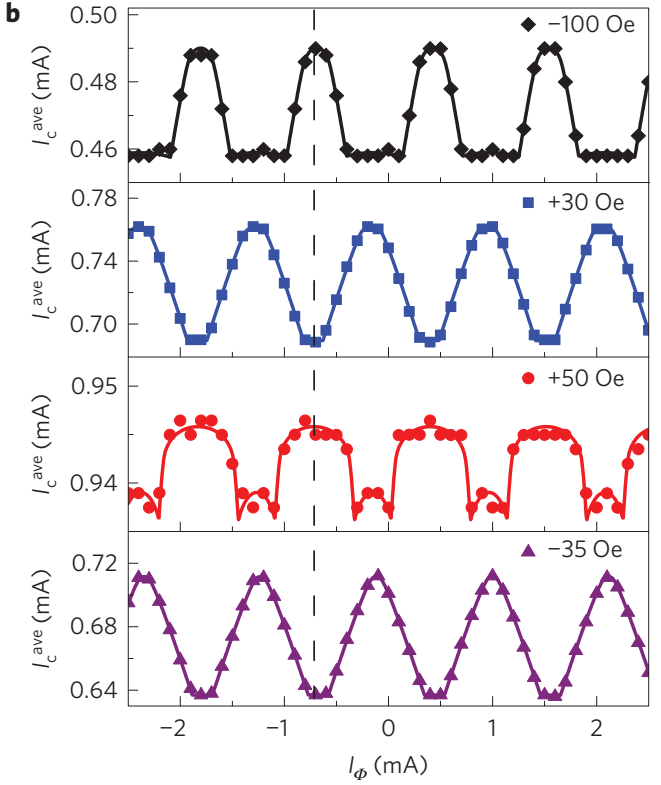

Figure $3 \mid I_{\mathrm{c}+}, I_{\mathrm{c}-}$ and $I_{\mathrm{c}}{ }^{\text {ave }}$ data with fits for all four magnetic states. a, Detailed plots of positive and negative SQUID critical currents, $I_{\mathrm{c}+}$ and $I_{\mathrm{c}-}$, versus flux-line current $I_{\Phi}$, for the four magnetic states implicated in Fig. 2. The states are labelled $(\pi-\pi),(0-\pi)$, and so on, according to the phase states of JJ-1 and $J J-2$, respectively. $I_{c+}$ and $I_{c-}$ both oscillate as a function of $I_{\Phi}$, but with a ratchet shape due to the finite and unequal geometrical inductances of the two arms of the SQUID loop. For each magnetic state, the two curves are shifted with respect to each other in opposite directions by amounts that depend on the individual critical currents, $I_{c 1}$ and $I_{c 2}$, of the two Josephson junctions. These critical currents will change depending on whether the junction is in the 0 or $\pi$ state. The solid lines are the result of least-squares fits to the data using the asymmetric SQUID model shown in Fig. 1c, as described in Methods and Supplementary Methods. b, Plot of average critical current, $I_{\mathrm{C}}{ }^{\mathrm{ave}}=\left(I_{\mathrm{c}+}-I_{\mathrm{c}-}\right) / 2$ versus $I_{\Phi}$, for the same four magnetic states represented in $\mathbf{a}$. The solid lines are derived from the fits in $\mathbf{a}$. Whereas the shapes of the $I_{\mathrm{c}}{ }^{\text {ave }}$ curves depend on the alignment between the $I_{\mathrm{c}+}$ and $I_{\mathrm{c}-}$ curves, the positions of the maximum and minima in $I_{c}$ ave are immune to the shifts in $I_{c+}$ and $I_{c-}$. This figure shows schematically the $\pi$ phase shifts in the $(0-\pi)$ and $(\pi-0)$ states relative to the $(\pi-\pi)$ and $(0-0)$ states. The analysis presented in Methods provides unambiguous proof of the $\pi$ phase shifts.

asymmetries in the inductances of the two arms of the loop and in the critical currents of the two junctions.

A simple model of an asymmetric SQUID is shown in Fig. 1c, where $L_{1}$ and $L_{2}$ are the effective inductances of the two arms of the SQUID loop and $I_{1}$ and $I_{2}$ are the currents through each $\operatorname{arm}^{22,23}$. Our SQUIDs have an inductance asymmetry, that is, $L_{1} \neq L_{2}$, because the current paths through the two sides of the SQUID have different lengths (see Fig. 1b). Our SQUIDs also have an asymmetry in the junction critical currents because the critical current is different when a junction is in the 0 versus the $\pi$ state. Asymmetries in the SQUID loop inductances and in the critical currents of the two junctions cause horizontal shifts of the $I_{c+}\left(I_{\Phi}\right)$ and $I_{c-}\left(I_{\Phi}\right)$ data in opposite directions, which change when the critical current in one of the junctions changes. One can remove those shifts from the data by plotting the average magnitude of the critical current, $I_{\mathrm{c}}{ }^{\text {ave }}=\left(I_{\mathrm{c}+}-I_{\mathrm{c}-}\right) / 2$ versus $I_{\Phi}$, as shown in Fig. $3 \mathrm{~b}$ for the four magnetic states represented in Fig. 3a. The $I_{\mathrm{c}}^{\text {ave }}\left(I_{\Phi}\right)$ curves have a variety of shapes depending on how much the $I_{\mathrm{c}+}\left(I_{\Phi}\right)$ and $I_{c-}\left(I_{\Phi}\right)$ curves in Fig. 3a are shifted with respect to each other. Regardless of the shapes, Fig. $3 \mathrm{~b}$ shows that the locations of the minima and maxima in $I_{\mathrm{c}}$ ave $\left(I_{\Phi}\right)$ line up with each other, with phase shifts of $\pi$ between successive curves. Figure $3 \mathrm{a}$ also shows independent fits to the $I_{c+}$ and $I_{c-}$ data, described under Methods and in the Supplementary Methods, which confirm a $\pi$ phase shift between each magnetic state. This demonstrates that we have been able to successfully control the phase of our junctions as proposed above.

The results represented in Fig. 3 are reproducible on repeating the whole major loop. In addition, one can obtain 'minor loop' data after initialization by keeping $H_{\text {in }}$ between +30 Oe and $-35 \mathrm{Oe}$, so that only the free layer of JJ-1 switches its state. We have obtained similar minor loop data from several different devices; the best major loop data were obtained in the device shown here.
In conclusion, we have demonstrated unequivocally a Josephson junction whose ground state can be switched between the 0-state and $\pi$-state by reversing the magnetization direction of one magnetic layer contained within the junction. Transitions between these states were verified by detecting the additional phase of the $\pi$-state junction within a d.c. SQUID. Phase-controllable Josephson junctions have applications in superconducting electronics based on single-flux-quantum logic. The most obvious application is in superconducting memory ${ }^{14-17}$. A single S/F/S Josephson junction with controllable critical current amplitude could function as a superconducting memory cell, but one must find a way to address such a memory cell when it is embedded in a large memory array, and the speed at which the junction switches into the voltage state after the 'read' current is applied is limited by the small $I_{\mathrm{c}} R_{\mathrm{N}}$ product of the junction. (S/F/S Josephson junctions with larger $I_{\mathrm{c}} R_{\mathrm{N}}$ product have been demonstrated ${ }^{24}$, but not with two ferromagnetic layers in a spin-valve configuration.) A solution to both problems is a memory cell based on a SQUID with a phase-controllable Josephson junction $^{11}$, such as the one shown schematically in Fig. 4 (ref. 10). In that cell, the $\mathrm{S} / \mathrm{F} / \mathrm{S}$ junction serves as a passive phase shifter; it has larger critical current than the two S/I/S junctions, so it stays in the supercurrent state during the read operation. The two $\mathrm{S} / \mathrm{I} / \mathrm{S}$ junctions have smaller $I_{\mathrm{c}}$ but large $I_{\mathrm{c}} R_{\mathrm{N}}$ product, hence they respond quickly to the read current. The state of the memory cell is determined by the critical current of the whole SQUID, which is large when the phase shifter is in the 0-state and small when it is in the $\pi$-state. A scheme for addressing individual SQUIDbased memory cells embedded in a large memory array has been proposed $^{25}$. Aside from memory applications, there are other singleflux-quantum circuits that already benefit from the use of fixedphase $\pi$-junctions $s^{7,9,10}$. One can now start to envisage new types of superconducting circuits using switchable $0-\pi$ junctions such as 


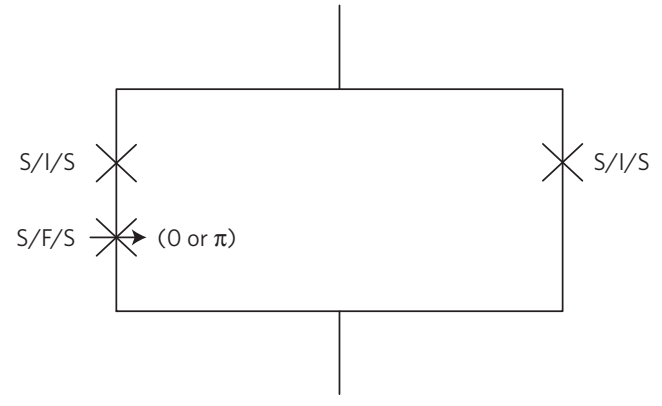

Figure 4 | Use of a controllable $0-\pi$ junction in a memory cell. This SQUID-based memory cell has high critical current when the controllable $\mathrm{S} / \mathrm{F} / \mathrm{S}$ junction is in the 0 -state and low critical current in the $\pi$-state. If the critical current of the S/F/S junction is larger than those of the two $S / I / S$ junctions, then only the latter switch into the voltage state when the read current is applied, whereas the $\mathrm{S} / \mathrm{F} / \mathrm{S}$ junction acts as a passive phase shifter ${ }^{10}$. The read time of the memory cell, $\tau_{\text {read }} \approx h / e l_{C} R_{N}$, is then determined by the faster $\mathrm{S} / \mathrm{I} / \mathrm{S}$ junctions with higher $I_{\mathrm{C}} R_{\mathrm{N}}$ product. Methods to address a single cell in a memory array are discussed in refs 11,25

the ones described here. This should open up new horizons in the nascent field of 'superconducting spintronics ${ }^{26}$.

\section{Methods}

Methods and any associated references are available in the online version of the paper.

\section{Received 19 October 2015; accepted 1 February 2016;} published online 14 March 2016

\section{References}

1. Buzdin, A. I., Bulaevskii, L. N. \& Panyukov, S. V. Critical-current oscillations as a function of the exchange field and thickness of the ferromagnetic metal (F) in a S-F-S Josephson junction. JETP Lett. 35, 178-180 (1982).

2. Ryazanov, V. V. et al. Coupling of two superconductors through a ferromagnet: evidence for a $\pi$ junction. Phys. Rev. Lett. 86, 2427-2430 (2001).

3. Kontos, T. et al. Josephson junction through a thin ferromagnetic layer: negative coupling. Phys. Rev. Lett. 89, 137007 (2002)

4. Terzioglu, E. \& Beasley, M. R. Complementary Josephson junction devices and circuits: a possible new approach to superconducting electronics. IEEE Trans. Appl. Supercond. 8, 48-53 (1998).

5. Ioffe, L. B., Geshkenbein, V. B., Feigel'man, M. V., Fauchere, A. L. \& Blatter, G. Environmentally decoupled $s d s$-wave Josephson junctions for quantum computing. Nature 398, 679-681 (1999).

6. Blatter, G., Geshkenbein, V. B. \& Ioffe, L. B. Design aspects of superconducting-phase quantum bits. Phys. Rev. B 63, 174511 (2001).

7. Ustinov, A. V. \& Kaplunenko, V. K. Rapid single-flux quantum logic using $\pi$-shifters. J. Appl. Phys. 94, 5405-5407 (2003).

8. Yamashita, T., Tanikawa, K., Takahashi, S. \& Maekawa, S. Superconducting $\pi$ qubit with a ferromagnetic Josephson junction. Phys. Rev. Lett. 95, 097001 (2005).

9. Khabipov, M. I. et al. A single flux quantum circuit with a ferromagnet-based Josephson $\pi$-junction. Supercond. Sci. Technol. 23, 045032 (2010).

10. Feofanov, A. K. et al. Implementation of superconductor/ferromagnet/superconductor $\pi$-shifters in superconducting digital and quantum circuits. Nature Phys. 6, 593-597 (2010).
11. Herr, A. Y. \& Herr, Q. P. Josephson magnetic random access memory system and method. US Patent 8,270,209 (2012).

12. Holmes, D. S., Ripple, A. L. \& Manheimer, M. A. Energy-efficient superconducting computing - power budgets and requirements. IEEE Trans. Appl. Supercond. 23, 1701610 (2013).

13. Demler, E. A., Arnold, A. B. \& Beasley, M. R. Superconducting proximity effects in magnetic metals. Phys. Rev. B 55, 15174-15182 (1997).

14. Bell, C. et al. Controllable Josephson current through a pseudospin-valve structure. Appl. Phys. Lett. 84, 1153-1155 (2004)

15. Baek, B., Rippard, W. H., Benz, S. P., Russek, S. E. \& Dresselhaus, P. D. Hybrid superconducting-magnetic memory device using competing order parameters. Nature Commun. 5, 3888 (2014)

16. Qader, M. A. E. et al. Switching at small magnetic fields in Josephson junctions fabricated with ferromagnetic barrier layers. Appl. Phys. Lett. 104, 022602 (2014)

17. Baek, B. et al. Spin-transfer torque switching in nanopillar superconducting-magnetic hybrid Joseph junctions. Phys. Rev. Appl. 3, 011001 (2015).

18. Robinson, J. W. A., Piano, S., Burnell, G., Bell, C. \& Blamire, M. G. Critical current oscillations in strong ferromagnetic $\pi$ junctions. Phys. Rev. Lett. 97, 177003 (2006)

19. Blum, Y., Tsukernik, A., Karpovski, M. \& Palevski, A. Oscillations of the superconducting critical current in $\mathrm{Nb}-\mathrm{Cu}-\mathrm{Ni}-\mathrm{Cu}-\mathrm{Nb}$ junctions. Phys. Rev. Lett. 89, 187004 (2002).

20. Klose, C. et al. Optimization of spin-triplet supercurrent in ferromagnetic Josephson junctions. Phys. Rev. Lett. 108, 127002 (2012).

21. Barone, A. \& Paterno, G. Physics and Applications of the Josephson Effect (Wiley, 1982).

22. Chesca, B., Kleiner, R. \& Koelle, D. in The SQUID Handbook Vol. 1 (eds Clarke, J. \& Braginski, A. I.) Ch. 2 (WILEY-VCH Verlag GmbH, 2004).

23. Tesche, C. D. \& Clarke, J. Dc SQUID: noise and optimization. J. Low Temp. Phys. 29, 301-331 (1977).

24. Larkin, T. I. et al. Ferromagnetic Josephson switching device with high characteristic voltage. Appl. Phys. Lett. 100, 222601 (2012).

25. Herr, A. Y., Herr, Q. P. \& Naaman, O. Phase hysteretic magnetic Josephson junction memory cell. US Patent Application 2015/0094207 (2015).

26. Linder, J. \& Robinson, J. W. A. Superconducting spintronics. Nature Phys. 11, 307-315 (2015)

\section{Acknowledgements}

We thank A. Herr, R. Kleiner, A. Miklich, N. Newman and N. Rizzo for helpful discussions, J. Willard for performing the FastHenry simulations, and B. Bi for help with fabrication using the Keck Microfabrication Facility. Preliminary work by Y.W. was supported by DOE-BES under grant \#DE-FG02-06ER46341. This research is supported by the Office of the Director of National Intelligence (ODNI), Intelligence Advanced Research Projects Activity (IARPA), via US Army Research Office contract W911NF-14-C-0115. The views and conclusions contained herein are those of the authors and should not be interpreted as necessarily representing the official policies or endorsements, either expressed or implied, of the ODNI, IARPA, or the US Government

\section{Author contributions}

E.C.G. and B.M.N. contributed equally to this work. The samples were designed by N.O.B., Y.W. and E.C.G. and fabricated by E.C.G. with significant help from B.M.N. and R.L. The data were taken by B.M.N. using apparatus designed by W.P.P.Jr and software written by Y.W. and E.C.G. The data were analysed by N.O.B., W.P.P.Jr, D.L.M. and J.A.G. The paper was written by N.O.B. and B.M.N. with help from W.P.P.Jr and input from all authors. The study was conceived by N.O.B. and W.P.P.Jr.

\section{Additional information}

Supplementary information is available in the online version of the paper. Reprints and permissions information is available online at www.nature.com/reprints. Correspondence and requests for materials should be addressed to N.O.B.

\section{Competing financial interests}

The authors declare no competing financial interests. 


\section{Methods}

Materials. Ni was chosen as the fixed layer material on the basis of earlier work ${ }^{20}$ showing that Ni layers of thickness 1.0 or $1.5 \mathrm{~nm}$ magnetize in applied fields of 1,000-2,000 Oe. Magnetometry measurements of $\mathrm{Cu} / \mathrm{Ni}$ multilayers show that such thin Ni films surrounded by $\mathrm{Cu}$ on both sides have magnetization

$M \approx 420$ e.m.u. $\mathrm{cm}^{-3}$, magnetically 'dead layers' of total thickness $\approx 0.4 \mathrm{~nm}$, and a Curie temperature slightly above room temperature for $d_{\mathrm{Ni}}=1.0 \mathrm{~nm}$, and well above room temperature for $d_{\mathrm{Ni}}=1.5 \mathrm{~nm}$ (data taken by C. Klose, unpublished Bachelor's thesis). Permalloy was chosen as the free layer material based on ongoing work in our lab showing good single-domain switching of NiFe nanomagnets embedded inside $\mathrm{Nb} / \mathrm{Cu} / \mathrm{NiFe} / \mathrm{Cu} / \mathrm{Nb}$ Josephson junctions (J.A.G., M. A. Khasawneh, B.M.N., E.C.G., R.L., W.P.P.Jr, and N.O.B., in preparation). The nominal sputtering target concentration is $\mathrm{Ni}_{0.80} \mathrm{Fe}_{0.20}$, but analysis of thick films by energy dispersive $\mathrm{x}$-ray spectroscopy gives $\mathrm{Ni}_{0.84} \mathrm{Fe}_{0.16}$. Data of critical current versus magnetic field (Fraunhofer patterns) provide a rough estimate of the NiFe magnetic moment in the junction. Fits to the data as a function of NiFe thickness then provide estimates of the NiFe magnetization, $M \approx 800$ e.m.u. $\mathrm{cm}^{-3}$, and dead-layer thickness of $0.1 \mathrm{~nm}$. In comparison, NiFe films surrounded by $\mathrm{Nb}$ show a dead-layer thickness of about $0.5 \mathrm{~nm}$ (ref. 18). We have not measured the Curie temperature of 1.5 -nm-thick NiFe films, but it is certainly well above room temperature.

Sample fabrication. The Josephson junctions and SQUIDs used in this work are fabricated using ultrahigh-vacuum sputtering deposition and standard microfabrication techniques, including photolithography, e-beam lithography, and ion milling. The bottom wiring layer is a $[\mathrm{Nb} / \mathrm{Al}]$ multilayer chosen to have lower surface roughness than pure $\mathrm{Nb}$, thereby improving the magnetic switching properties of the soft magnetic materials in the junctions ${ }^{27}$. This bottom wiring layer and all of the ferromagnetic layers inside the junction, including a 20 -nm top layer of $\mathrm{Nb}$ and a final layer of Au to prevent oxidation, are deposited in a single sputtering run without breaking vacuum, to ensure high-quality interfaces. The sputtering chamber is equipped with a liquid-nitrogen-cooled Meissner trap to reduce the partial pressure of water vapour. The base pressure of the sputtering chamber before deposition is $<2 \times 10^{-8}$ torr, whereas the Ar pressure during sputtering is about 2 mtorr. Measurements of the area-resistance product in the normal state yield consistent values of $\mathrm{AR}_{\mathrm{N}} \approx 6 \mathrm{f} \Omega-\mathrm{m}^{2}$ for many junctions of different sizes-an indicator of the reproducible high quality of the interfaces. The bottom layer is patterned using photolithography and the lift-off process. The junctions are patterned by electron-beam lithography and Ar ion milling, using the negative e-beam resist ma-N2401 as the ion mill mask. The junctions are sufficiently small, with an area of $0.5 \mu \mathrm{m}^{2}$ to ensure that the NiFe layers are single domain ${ }^{28}$. After milling, $\mathrm{SiO}_{x}$ layer is deposited by thermal evaporation to electrically isolate the junction and the bottom wiring layer from the top wiring layer. Finally, the top $\mathrm{Nb}$ wiring layer is deposited by sputtering, again using photolithography and lift-off to define the pitch-fork-like pattern seen in Fig. $1 \mathrm{~b}$. The final SQUID loop has inner dimensions of $10 \mu \mathrm{m} \times 10 \mu \mathrm{m}$ with 5 - $\mu \mathrm{m}$-wide strips.

Measurement. The measurements reported here were performed at $4.2 \mathrm{~K}$ with the samples immersed in a liquid helium dewar equipped with a Cryoperm magnetic shield. The sample dip-stick is equipped with a commercial rf SQUID that is used in a self-balancing potentiometer circuit to measure the voltage across the sample SQUID, and a superconducting solenoid to apply uniform fields in the plane of the sample. The measurement current through the sample SQUID is provided by a battery-powered ultralow-noise programmable current source. The flux current, $I_{\phi}$, is provided by a Yokogawa programmable voltage source and a $1 \mathrm{k} \Omega$ resistor. $I-V$ curves are obtained by sweeping the measurement current $I$ from 0 to a value just above where the sample goes into the voltage state, to determine $I_{c+}$, and then sweeping from 0 in the negative direction to a value just beyond $I_{c-}$.

Initialization of the Ni magnetizations requires applying a large in-plane field $\left|H_{\text {in }}\right|=2,600 \mathrm{Oe}$. After returning the field to zero, we lift the dip-stick several inches until the sample is just above the liquid helium level and the $\mathrm{Nb}$ wiring layers are no longer superconducting, to remove any trapped magnetic flux from the superconducting layers. The sample is then lowered back into the liquid helium and left there for the remainder of the run. The maximum field applied after that is $\pm 100 \mathrm{Oe}$, which is small enough not to induce any trapped flux in the $\mathrm{Nb}$ lines.

Data analysis. The standard model for an asymmetric dc SQUID is shown in Fig. 1c. The SQUID is characterized by the four parameters: $L_{1}, L_{2}, I_{\mathrm{c} 1}$ and $I_{\mathrm{c} 2}$, which are the effective inductances of the two arms and the critical currents of the two Josephson junctions. $L_{1}$ and $L_{2}$ are simply related to the geometric inductances of the two arms if the mutual inductance between them is properly taken into account ${ }^{23}$. On the basis of the geometry shown in Fig. 1b, we expect that $L_{2}>L_{1}$. In our samples $L_{1}$ and $L_{2}$ are fixed, whereas $I_{\mathrm{c} 1}$ and $I_{\mathrm{c} 2}$ change depending on whether the corresponding junction is in the 0 or $\pi$ state. The externally applied flux is $\Phi$, defined as positive when the magnetic field $\mathbf{B}$ points out of the page. In addition, the SQUID acquires an extra phase shift of $\pi$, or equivalently an extra flux of $\Phi_{0} / 2$, when one of the two Josephson junctions is in the $\pi$ state. When both junctions are in the $\pi$ state, the two additional phase shifts cancel.

Figure $3 \mathrm{~b}$ shows the average critical current, $I_{\mathrm{c}}^{\text {ave }}=\left(I_{\mathrm{c}+}-I_{\mathrm{c}-}\right) / 2$, versus current through the flux line, $I_{\phi}$. The locations of the maxima and minima in $I_{c}^{\text {ave }}\left(I_{\phi}\right)$ line up with each other, with phase shifts of $\pi$ between successive curves. Figure $3 b$ alone is not sufficient to deduce that the SQUID has acquired an extra phase shift of $\pi$ each time the state of one junction changed, because apparent $\pi$ shifts in the $I_{\mathrm{c}}$ ave $\left(I_{\Phi}\right)$ curves can arise purely from changes in $I_{\mathrm{c} 1}$ or $I_{\mathrm{c} 2}$. One can detect the presence of $\pi$ phase shifts by analysing the shifts in the peak positions of the $I_{c+}$ and $I_{c-}$ curves when the SQUID transitions from state to state, as shown by the following argument. The current reaches its maximum value, $I_{\mathrm{c}}=I_{\mathrm{c} 1}+I_{\mathrm{c} 2}$, when the phase drop across each junction is $\pi / 2$, so that the currents $I_{1}$ and $I_{2}$ through the two arms of the SQUID equal $I_{\mathrm{c} 1}$ and $I_{\mathrm{c} 2}$, respectively. Those currents induce a flux through the SQUID loop, $\Phi_{\text {self }}{ }^{+}=L_{1} I_{\mathrm{cl}}-L_{2} I_{\mathrm{c} 2}$. That must be balanced by the externally applied flux, so the peak in $I_{c+}$ occurs at a flux of

$\Phi_{\text {peak }}{ }^{+}=-\Phi_{\text {self }}{ }^{+}=L_{2} I_{\mathrm{c} 2}-L_{1} I_{\mathrm{c} 1}$. The maximum negative critical current occurs at flux $\Phi_{\text {peak }}{ }^{-}=-\Phi_{\text {peak }}{ }^{+}$, hence the peaks in $I_{c+}(\Phi)$ and $I_{c-}(\Phi)$ are shifted with respect to each other by $\Delta \Phi_{\text {peak }} \equiv \Phi_{\text {peak }}{ }^{+}-\Phi_{\text {peak }}{ }^{-}=2\left(L_{2} I_{\mathrm{c} 2}-L_{1} I_{\mathrm{c1}}\right)$. Because the $I_{c}(\Phi)$ curves are periodic, $\Delta \Phi_{\text {peak }}$ can be determined only modulo $\Phi_{0}$. That means that the centre of the pattern - that is, the point half-way between an $I_{c+}$ peak and its corresponding $I_{c-}$ peak - can be determined only modulo $\Phi_{0} / 2$. Fortunately, it is possible to determine which of the possible values for $\Delta \Phi_{\text {peak }}$ is the physical value, by analysing the changes in $\Delta \Phi_{\text {peak }}$ when the critical current of one of the junctions changes. If JJ- 1 changes its critical current by an amount $\delta I_{\mathrm{c} 1}$ whereas $I_{\mathrm{c} 2}$ remains unchanged, then the peak separation will change by $\delta\left(\Delta \Phi_{\text {peak }}\right)=-2 L_{1} \delta I_{\mathrm{cl}}$. Turning the argument around, one can extract the inductance $L_{1}$ from the transition using $L_{1}=-\delta\left(\Delta \Phi_{\text {peak }}\right) / 2 \delta I_{\mathrm{cl}}$. Similarly, if JJ-2 changes its critical current by an amount $\delta I_{\mathrm{c} 2}$ whereas $I_{\mathrm{cl}}$ remains unchanged, then the peak separation will change by $\delta\left(\Delta \Phi_{\text {peak }}\right)=+2 L_{2} \delta I_{\mathrm{c} 2}$. What one finds is that, if one takes the wrong value of $\delta\left(\Delta \Phi_{\text {peak }}\right)$ for a transition, then the value of $L_{1}$ or $L_{2}$ extracted from the transition is unphysical. Our data set provides four transitions; the transitions from the $(\pi-\pi)$ state to the $(0-\pi)$ state and from the $(0-0)$ state to the $(\pi-0)$ state allow us to extract $L_{1}$, because only $I_{\mathrm{cl}}$ changes, whereas the transitions from the $(0-\pi)$ state to the $(0-0)$ state and from the $(\pi-0)$ state to the $(\pi-\pi)$ state allow us to extract $L_{2}$, because only $I_{\mathrm{c} 2}$ changes. Only one set of phase shifts produces a consistent set of values, $L_{1}=6.0 \pm 0.5 \mathrm{pH}$ and $L_{2}=11.7 \pm 0.1 \mathrm{pH}$. These values are in agreement with the results of simulations of our SQUID geometry using the FastHenry software, which produced values in the range of 6-7 $\mathrm{pH}$ for $L_{1}$ and 13 $\mathrm{pH}$ for $L_{2}$, with variations of about $1 \mathrm{pH}$ for each depending on the type of mesh used in the simulation. Different choices of $\delta\left(\Delta \Phi_{\text {peak }}\right)$ that do not include the $\pi$ shifts yield values of $L_{1}$ and $L_{2}$ that differ in either direction by about $5 \mathrm{pH}$; those values are not only inconsistent with simulations of our geometry, but more importantly they are inconsistent with the observed depth of the $I_{\mathrm{c}}(\Phi)$ oscillations, which must be approximately equal to $\Phi_{0} / L$ (ref. 22). The phase shifts deduced from our analysis confirm that the SQUID does indeed acquire a phase shift of $\pi$ each time the system transitions between successive states in the sequence.

Analysing the four SQUID transitions also provides us with values for $\delta I_{\mathrm{c} 1}$ and $\delta I_{\mathrm{c} 2}$, but not values of the critical currents for each junction in each magnetic state. To estimate the latter, one can assume that the ratios of critical current densities between the 0 and $\pi$ states in both junctions are equal. That assumption implies that $I_{\mathrm{c} 1}{ }^{0} / I_{\mathrm{c} 1}{ }^{\pi}=I_{\mathrm{c} 2}{ }^{0} / I_{\mathrm{c} 2}{ }^{\pi}$, but allows the areas of the two junctions to differ. That analysis leads to the approximate values $I_{\mathrm{c} 1}{ }^{0}=560 \mu \mathrm{A}, I_{\mathrm{c} 1}{ }^{\pi}=290 \mu \mathrm{A}, I_{\mathrm{c} 2}{ }^{0}=420 \mu \mathrm{A}$ and $I_{\mathrm{c} 2}{ }^{\pi}=220 \mu \mathrm{A}$, with uncertainties of the order of $10 \mu \mathrm{A}$.

To obtain more accurate values of the SQUID parameters, we performed a nonlinear least-squares fit to the data of a numerical analysis of the asymmetric SQUID using the Mathematica software. The model is described by equations (2.64), (2.72) and (2.73) in ref. 22. Those equations use the following set of dimensionless variables to describe the SQUID. The total SQUID inductance is characterized by $\beta_{\mathrm{L}}=L I_{\mathrm{c}} / \Phi_{0}$, where $L=L_{1}+L_{2}$ is the total loop inductance of the SQUID and $I_{\mathrm{c}}=I_{\mathrm{c} 1}+I_{\mathrm{c} 2}$ is the maximum critical current of the SQUID. If $\beta_{\mathrm{L}} \ll 1$, $L_{1}=L_{2}$ and $I_{\mathrm{c} 1}=I_{\mathrm{c} 2}$, then the oscillations of critical current with respect to flux have the standard form, $I_{\mathrm{c}}(\Phi)=I_{\mathrm{c}}\left|\cos \left(2 \pi \Phi / \Phi_{0}\right)\right|$. If $\beta_{\mathrm{L}}>1$, as is the case with our SQUIDs, then the $I_{\mathrm{c}}(\Phi)$ oscillations do not extend to zero, but rather have an amplitude approximately equal to $\Phi_{0} / L$. The asymmetry in the inductances of the two arms is characterized by $\alpha_{\mathrm{L}}=\left(L_{2}-L_{1}\right) /\left(L_{2}+L_{1}\right)$. As $\alpha_{\mathrm{L}}$ increases,

$I_{c-}(\Phi) \neq-I_{c+}(\Phi)$, and the $I_{\mathrm{c}}(\Phi)$ oscillations become more asymmetric, taking on a 'ratchet shape', as shown by the data of Figs 2 and 3a. This ratchet shape leans to the right, indicating that $L_{2}>L_{1}$ or $\alpha_{\mathrm{L}}>0$. These relationships are expected on the basis of the geometry of the SQUID and direction of the currents shown in Fig. 1b. By changing the wiring of the SQUID so that the sample current exits out the opposite end of the bottom lead, we should observe that $L_{1}>L_{2}$ or $\alpha_{\mathrm{L}}<0$, and the $I_{\mathrm{c}+}(\Phi)$ curves appear as a ratchet leaning to the left. This has been confirmed experimentally. The asymmetry in the critical currents is characterized by $\alpha_{\mathrm{I}}=\left(I_{\mathrm{c} 2}-I_{\mathrm{c} 1}\right) /\left(I_{\mathrm{c} 2}+I_{\mathrm{c} 1}\right)$. As $\alpha_{1}$ deviates more from zero, the magnitudes of the slopes of both the rising and falling portions of the $I_{c}(\Phi)$ curves decrease, hence the depth of the $I_{\mathrm{c}}$ modulation decreases. In our samples, $\alpha_{\mathrm{L}}$ should remain the same for all four magnetic states, whereas $\beta_{\mathrm{L}}$ and $\alpha_{\mathrm{I}}$ change because the critical 
currents change. In addition to determining the shapes of the $I_{\mathrm{c}}(\Phi)$ curves, non-zero values of $\beta_{\mathrm{L}}, \alpha_{\mathrm{L}}$ and $\alpha_{\mathrm{I}}$ also shift the positions of the peaks in $I_{\mathrm{c}}(\Phi)$, as discussed above: $\Phi_{\text {peak }}{ }^{+}=\left(L_{2} I_{\mathrm{c} 2}-L_{1} I_{\mathrm{c} 1}\right)=\beta_{\mathrm{L}}\left(\alpha_{\mathrm{L}}+\alpha_{\mathrm{I}}\right) \Phi_{0} / 2$

To reduce the number of free parameters in the fits, we first determined the conversion from $I_{\Phi}$ to $\Phi$ by fitting the $I_{\mathrm{c}}^{\text {ave }}(\Phi)$ curves shown in Fig. $3 \mathrm{~b}$ with a simple Fourier series. For the $(0-\pi)$ and $(\pi-0)$ states, a single cosine wave fits the data well, whereas for the $(0-0)$ and $(\pi-\pi)$ states we used a cosine wave plus its second harmonic. From the Fourier series fits to the four data sets we determined that $I_{\Phi}=(1,115 \pm 2) \mu \mathrm{A} * \Phi / \Phi_{0}$. That conversion factor was then kept fixed in all the ensuing fits. The fitting procedure was as follows. For each magnetic state, the data for $I_{\mathrm{c}}^{+}\left(I_{\Phi}\right)$ and $I_{\mathrm{c}}^{-}\left(I_{\Phi}\right)$ were fitted simultaneously. The free parameters in each fit were $I_{\mathrm{c}+},\left|I_{\mathrm{c}-}\right|, \beta_{\mathrm{L}}, \alpha_{\mathrm{L}}, \alpha_{\mathrm{I}}$ and $\phi_{\text {shift }}$. Although we expected $I_{\mathrm{c}+}$ and $\left|I_{\mathrm{c}-}\right|$ to be equal to each other, the data exhibited small differences between $I_{c+}$ and $\left|I_{c-}\right|$, of order a few $\mu \mathrm{A}$. The last parameter is the shift of the centre of the pattern relative to zero flux, $\phi_{\text {shift }} \equiv \Phi_{\text {shift }} / \Phi_{0}$. The values of the dimensionless fitting parameters and the corresponding physical SQUID parameters extracted from the fits to all four magnetic states are shown in the Supplementary Methods. The independent fits to the four magnetic states produce remarkably consistent results for the values of the two SQUID inductances and the critical currents of the two junctions, each in two magnetic states. The physical values averaged over the fits are: $L_{1}=5.68 \pm 0.05 \mathrm{pH}$ $L_{2}=11.46 \pm 0.12 \mathrm{pH}, I_{\mathrm{cl}}{ }^{0}=565.9 \pm 1.4 \mu \mathrm{A}, I_{\mathrm{c} 1}{ }^{\pi}=292.8 \pm 1.2 \mu \mathrm{A}$, $I_{\mathrm{c} 2}{ }^{0}=419.5 \pm 0.2 \mu \mathrm{A}$ and $I_{\mathrm{c} 2}{ }^{\pi}=210 \pm 7 \mu \mathrm{A}$. Those values are very close to the preliminary values extracted from the analysis of the peak positions described above. Most importantly, the values of $\phi_{\text {shift }}$ confirm the additional phase shifts of $\pi$ that occur at each magnetic transition. More detail is provided in the Supplementary Methods

\section{References}

27. Wang, Y., Pratt, W. P. Jr \& Birge, N. O. Area-dependence of spin-triplet supercurrent in ferromagnetic Josephson junctions. Phys. Rev. B 85, 214522 (2012)

28. Niedzielski, B. M., Loloee, R., Pratt, W. P. Jr \& Birge, N. O. S/F/S Josephson junctions with single domain ferromagnets for memory applications. Supercond. Sci. Technol. 28, 085012 (2015). 